\title{
Processo markoviano de decisão para controle de admissão de conexão e alocação dinâmica de recursos em redes IEEE 802.16
}

\author{
Cynthia F. Leal, Solon V. de Carvalho, Adriana N. F. da Rosa, Carlos R. L. Francês, João C. W. A. Costa
}

\begin{abstract}
Resumo-Neste trabalho investigam-se políticas de controle de admissão de conexão e alocação dinâmica de recursos em redes IEEE 802.16. Em linhas gerais, o trabalho consiste na determinação de uma política ótima que maximiza a utilização de recursos e que permite o tratamento diferenciado dos fluxos de tráfego objetivando atender os diferentes requisitos de QoS das classes de serviço definidas no padrão IEEE 802.16. O problema é formulado como um processo markoviano de decisão (MDP) e um algoritmo de iteração de valores é utilizado para obtenção da política de alocação ótima. Resultados numéricos são apresentados.
\end{abstract}

Palavras-Chave- IEEE 802.16, Controle de Admissão de Conexão (CAC), Processo Markoviano de Decisão.

Abstract - This paper outlines policies for call admission control and dynamic resource assignment in IEEE 802.16 networks. In general, the purpose of work the determination of an optimal policy that maximizes the resources utilization and allowing the differentiated treatment of traffic flows aiming to meet the different requirements of QoS class of service defined in IEEE 802.16 standard. The problem can be formulated as a Markov Decision Process (MDP) and an algorithm of iteration of values is used to obtain the optimal allocation policy. Numerical results are presented.

Keywords - IEEE 802.16, Call Admission Control (CAC), Markov Decision Process.

\section{INTRODUÇÃO}

O padrão IEEE 802.16[1][2] tem como proposta inicial disponibilizar uma solução sem fio para o problema de acesso de última milha em redes metropolitanas, possibilitando a cobertura de distâncias maiores sem a necessidade de investimento em infra-estrutura de alto custo como ocorre em redes de acesso banda larga cabeada reduzindo desta forma, o custo de implantação e do tempo necessário para se conectar residências e escritórios aos backbones das linhas de comunicação. O IEEE 802.16 fornece a especificação da camada física (PHY) e camada de enlace (MAC) visando suprir as deficiências das redes sem fio no que se refere à extensão geográfica de alcance da rede e às taxas de transmissão de dados.

Cynthia F. Leal Adriana N. F. da Rosa, and João C. W A. Costa Laboratório de Eletromagnetismo Aplicado, Carlos R. L. Francês, Laboratório de Planejamento de Redes de Alto Desempenho, Universidade Federal do Pará, Belém, PA, E-mails: \{adrianan, cynthia, jweyl, rfrances\}@ufpa.br. Solon V. de Carvalho, Laboratório Associado de Computação e Matemática Aplicada, Instituto Nacional de Pesquisas Espaciais - INPE, São José dos Campos, SP, Brazil, E-mail: Solon@lac.inpe.br. Este Trabalho foi parcialmente financiado pelo CNPq e pela CAPES, projeto PROCAD número 0226050.
A camada de enlace (MAC) do padrão, definida como orientada a conexão, foi projetada para permitir o tratamento diferenciado dos fluxos de tráfego objetivando atender os diferentes requisitos de QoS de cada fluxo. Neste sentido, o padrão define cinco classes serviço:

- Unsolicited Grant Service (UGS): Suporta aplicações de tempo real com taxa de bits constante em intervalos periódicos. Esse é o caso, por exemplo, de VoIP sem supressão de silêncio.

- Extended Real-Time Polling Service (ertPS): A classe de serviço ertPS foi adicionado na ratificação do 802.16e[1] e suporta aplicações de tempo real com taxa de bits variável em intervalos periódicos e com restrições de atraso. Um exemplo de aplicações deste tipo é VoIP com supressão de silêncio.

- Real-Time Polling Service (rtPS): Suporta aplicações de tempo real com taxa variável de bits em intervalos periódicos. Como, por exemplo, tramissão de videos MPEG (Moving Pictures Experts Group).

- Non-Real-Time Polling Service (nrtPS): Foi desenvolvida pra suportar aplicações sem requisitos de tempo real com taxa variável bits em intervalos periódicos, como, por exemplo, o tráfego gerado por aplicações FTP, e-mail, entre outras.

- Best Effort (BE): Projetada para aplicações de melhor esforço com garantias mínimas de QoS. O serviço de melhor esforço é tipicamente oferecido para o tráfego gerado por navegação na Internet.

Além da definição de classes serviço existem outros três componentes que desempenham um papel fundamental para o provimento de QoS, são eles: o controle de admissão de conexão, o escalonamento de pacotes e o gerenciador de Buffer. O controle de admissão de conexão limita o número de conexões na rede permitindo que cada conexão tenha satisfeitos seus requisitos de QoS. O sistema necessita ainda definir uma ordem de transmissão para os vários pacotes de forma a manter a qualidade de serviço estabelecida para cada conexão seja satisfeita, a esse processo chamamos escalonamento de pacotes. Comparando, enquanto o controle de admissão de conexão cuja decisão é feita baseada em requisitos à longo prazo 
(ex. probabilidade de bloqueio), o escalonamento de pacotes lida com requisitos instantâneos (ex. atraso). Outra forma de prover QoS é através do gerenciador de buffer que controla o tamanho do buffer e decide qual pacote descartar. Em suma, um controle de admissão de conexão ótimo, um escalonador de pacotes eficiente e um gerenciador de buffer justo são essenciais para proporcionar QoS em redes banda larga sem fio. Entretanto, nenhum deles é definido completamente pelo padrão 802.16 .

\section{DESCRIÇÃO DO PROBLEMA}

O controle de admissão conexão (CAC) é de suma importância em qualquer rede que permita provisão QoS para suas conexões, pois possibilita o controle da utilização dos recursos da rede. A principal função do CAC é decidir adequadamente se canal de comunicação deve ou não aceitar uma nova conexão. Caso a política do controle aceite um número excessivo de conexões o sistema não terá com garantir a QoS das conexões existentes, entretanto caso a política de controle admita um número muito pequeno de conexões, ou seja, rejeite muitas conexões, pode ocorrer um desperdício dos recursos da rede. Dentre as estratégias utilizadas para solucionar o problema de admissão destacam-se a complete sharing (CS) e a complete partitioning (CP)[3]. Em linhas gerais, na política CS permite um acesso igualitário dos recursos da rede. Esta estratégia resulta em máxima utilização da largura de banda disponível, especialmente redes com muito tráfego. Entretanto, o tratamento igualitário de conexões com prioridade diferentes pode trazer conseqüências severas quando conexões de uma classe necessitam de muito menos recurso que outras. Nesta situação poderá ser desejável rejeitar conexões com baixo requisito de largura de banda para aumentar a probabilidade de aceitar uma futura conexão maior necessidade de largura de banda. A política $\mathrm{CP}$ atribui a cada classe uma faixa da largura de banda total da rede, que não pode ser utilizada por conexões de outras classes. Sendo assim, ela suporta serviços diferenciados e controle da probabilidade de bloqueio das classes. Entretanto, a política $\mathrm{CP}$ não maximiza a utilização de todos os recursos disponíveis.

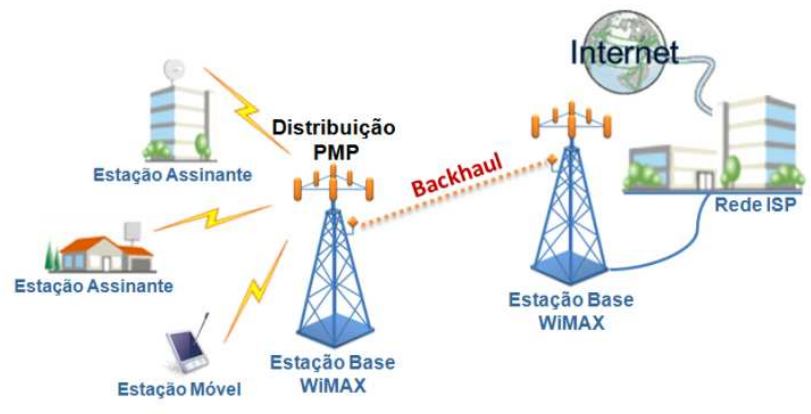

Fig. 1 Rede Wimax PMP.

O controle de admissão de conexão em uma rede IEEE 802.16 deve permitir tanto o tratamento diferenciado das classes de serviço como objetivar a maximização da utilização dos recursos da rede. Neste contexto, no presente trabalho propõe-se uma solução para este problema através da modelagem de um Processo Markoviano de Decisão (PMD) [4] cujo objetivo é obter uma política ótima de admissão que maximize a utilização da largura de banda da rede.

Para o controle de admissão proposto utiliza-se o conceito de degradação de largura de banda proposto por [5], o qual é baseado nos requisitos diferenciados de largura de banda das classes de serviço do IEEE 802.16, por exemplo, conexões das classes ertPS, rtPS e nrtPS possuem uma taxa máxima e mínima de largura de banda. O padrão IEEE 802.16 especifica que deve ser garantido pelo menos uma largura de banda mínima para que este parâmetro QoS seja satisfeito, isto permite que o excedente de banda da conexão em questão possa ser "emprestado" para ser utilizado em novas conexões. É interessante notar que não pode haver "empréstimo" de largura de banda de conexões da classe UGS, pois estas necessitam de largura de banda constante, conforme estabelecido pelo padrão IEEE 802.16. Vale ressaltar ainda que o conceito de degradação apresentado em [5] considera que somente conexões da classe nrtPS podem ser degradadas, porém neste trabalho esse conceito será estendido para as conexões da classes ertPS e rtPS, objetivando obter maior utilização da rede.

Assim, para a modelagem do problema considera-se uma rede IEEE 802.16 com topologia Ponto Multiponto (PMP), conforme mostrada na Figura 1. O tráfego na estação base (BS) é divido em três classes de serviços. A classe 1, corresponde às conexões da classe de serviço UGS, a classe 2, corresponde às conexões da classes de serviço ertPS e rtPS, agrupou-se essas duas classes devido serem equivalentes em termo de requisitos de banda. Finalmente, a classe 3 do modelo está associada à classe nrtPS. Não se considera a classe de serviço BE na modelagem, pois se assume que a mesma é sempre admitida pela $\mathrm{BS}$, porém não há reserva de recurso no momento da admissão.

Assume-se que a largura de banda requerida pelas conexões da classe 1 é $b_{1}$, devendo ser satisfeita para que garantir os requisitos sua QoS. As taxas de transmissão das conexões da classe 2 e 3 podem variar na faixa $\left[b_{j}^{\text {min }}, b_{j}^{\text {max }}\right]$, onde $b_{j}^{\text {min }}$ e $b_{j}^{\text {max }}$ são a banda máxima e mínima para conexões da classe $j$, onde $j=(2,3)$. Quando existe banda suficiente disponível no sistema (baixo número de conexões ativas), as conexões podem transmitir a altas taxas, porém conforme o número de conexões no sistema aumenta, as conexões das classes 2 e 3 existentes podem dispensar certa quantidade de largura de banda com o objetivo permitir que um maior número de novas conexões seja aceito, maximizando desta forma a utilização da rede. Entretanto, a classe 2 só deve ser degradada com o intuito de aceitar novas conexões desta mesma classe ou da classe 1 , já a classe 3 pode ser degrada 
para que seja possível aceitar um nova conexão de qualquer classe.

A degradação é feita em passos onde $\delta$ é a quantia de largura de banda degradada para cada passo de degradação das conexões da classe 2 e 3 . Considera-se que todas as conexões de uma classe devem manter o mesmo nível de degradação, sendo $l_{j}^{n}$ o nível de degradação da classe $j$. Assim, a largura de banda reservada para cada conexão é $b_{j}^{\text {max }}-l_{j}^{n} \delta$ que satisfaz $b_{j}^{\text {max }}-l_{j}^{n} b_{j}^{\text {min }} \leq b_{j}^{\text {min }}$. O passo máximo de degradação é definido por $l_{j}^{\max }=\frac{b_{j}^{\text {max }}-b_{j}^{\min }}{\delta}$, onde $j=(2,3)$. O processo de degradação da largura de banda da classe 2 e 3 pode, potencialmente, terminar por servir as conexões destas classe somente a banda mínima de cada uma. Para evitar isso se deve fazer uma redistribuição da banda sempre que houver disponibilidade para tal.

\section{FOMULAÇÃo DO PROCESSO MARKOVIANO DE DECISÃO}

A formulação do PMD para o problema inclui principalmente três passos:

- Primeiro, define-se espaço de estados finito para todas as conexões ativas na rede.

- Segundo, especificam-se as ações e a dinâmica dos estados do PMD.

- Terceiro, especificam-se o critério de performance para maximização do retorno médio a longo prazo.

\subsection{Espaço de estados.}

Para o modelo de controle de admissão assume-se que, o processo de chegada de novas conexões das classes 1, 2, 3 é uma distribuição de Poisson com taxas $\lambda_{1}, \lambda_{2}$ e $\lambda_{3}$, respectivamente. Os tempos de serviço das conexões das classes 1, 2 e 3 são exponencialmente distribuídos com médias $1 / \mu_{1}, 1 / \mu_{2}$ e $1 / \mu_{3}$, respectivamente. Cada BS é modelada como uma cadeia de Markov de tempo continuo cujo estado é representado por $s=\left(n_{1}, n_{2}, n_{3}, l_{2}^{n}, l_{3}^{n}, e v\right)$, onde $n_{1}, n_{2}$ e $n_{3}$ são o número de conexões ativa da classe 1,2 e 3 , respectivamente; $l_{j}^{n}$ é o nível de degradação das conexões da classe $j$ do sistema, onde $j=(2,3)$; e $e v$ representa a ocorrência de evento que pode ser:

○ $a_{1}, a_{2}, a_{3}$ : chegada de uma nova conexão da classe 1,2 e 3 , respectivamente.

○ $s_{1}, s_{2}, s_{3}$ : término de conexão da classe 1,2 e 3 , respectivamente. é:

Desta forma, o espaço de estados $S$ do modelo proposto

$$
\begin{gathered}
S=\left\{\left(n_{1}, n_{2}, n_{3}, l_{2}^{n}, l_{3}^{n}, e v\right) \mid n_{1} \geq 0, n_{2} \geq 0, n_{3} \geq 0,\right. \\
e v \in\left\{a_{1}, a_{2}, a_{3}, s_{1}, s_{2}, s_{3}\right\},
\end{gathered}
$$

$$
\begin{aligned}
& n_{1} b_{1}+n_{2}\left(b_{2}^{\max }-l_{3}^{n} \delta\right)+n_{3}\left(b_{3}^{\max }-l_{3}^{n} \delta\right) \leq B \\
& l_{3}^{n} \leq l_{3}^{\max }, \\
& l_{2}^{n} \leq l_{2}^{\max }, \\
& \text { se } n_{1}=0 \text { então e } v \neq s_{1}, \\
& \text { se } n_{2}=0 \text { então e } \neq \neq s_{2}, \\
& \text { se } n_{3}=0 \text { então e } v \neq s_{3},
\end{aligned}
$$

\subsection{Dinâmica dos estados.}

Por hipótese, o sistema é observado continuamente no tempo. A cada chegada de uma nova chamada, deve-se decidir sobre sua aceitação ou rejeição ( $A$ ou $R$ ). Uma nova chamada só poderá ser aceita quando houver largura de banda suficiente para alocá-la. A cada término de conexão, esta sai do sistema e nenhuma decisão é tomada, ou seja, deve-se adotar a ação $N$ (nenhuma ação).

Assim para cada estado $s=\left(n_{1}, n_{2}, n_{3}, l_{2}^{n}, l_{3}^{n}, e v\right) \in S$, as ações possíveis $\mathrm{A}(\mathrm{s})$ :

$$
A(s)=\left\{\begin{array}{l}
\{A, R\} \text { se }\left\{\begin{array}{l}
\left(e v=a_{1}\right) \wedge\left(\left(n_{1}+1\right) b_{1}+n_{2}\left(b_{2}^{\max }-l_{2}^{n} \delta\right)+n_{3}\left(b_{3}^{\max }-l_{3}^{n} \delta\right) \leq B\right) \vee \\
\left(e v=a_{2}\right) \wedge\left(n_{1} b_{1}+\left(n_{2}+1\right)\left(b_{2}^{\max }-l_{2}^{n} \delta\right)+n_{3}\left(b_{3}^{\max }-l_{3}^{n} \delta\right) \leq B\right) \vee \\
\left(e v=a_{3}\right) \wedge\left(n_{1} b_{1}+n_{2}\left(b_{2}^{\max }-l_{2}^{n} \delta\right)+\left(n_{3}+1\right)\left(b_{3}^{\max }-l_{3}^{n} \delta\right) \leq B\right)
\end{array}\right. \\
\{R\} s e\left\{\begin{array}{l}
\left(e v=a_{1}\right) \wedge\left(\left(n_{1}+1\right) b_{1}+n_{2}\left(b_{2}^{\max }-l_{2}^{n} \delta\right)+n_{3}\left(b_{3}^{\max }-l_{3}^{n} \delta\right)>B\right) \vee \\
\left(e v=a_{2}\right) \wedge\left(n_{1} b_{1}+\left(n_{2}+1\right)\left(b_{2}^{\max }-l_{2}^{n} \delta\right)+n_{3}\left(b_{3}^{\max }-l_{3}^{n} \delta\right)>B\right) \vee \\
\left(e v=a_{3}\right) \wedge\left(n_{1} b_{1}+n_{2}\left(b_{2}^{\max }-l_{2}^{n} \delta\right)+\left(n_{3}+1\right)\left(b_{3}^{\max }-l_{3}^{n} \delta\right)>B\right)
\end{array}\right. \\
\{N\} \text { se ev }=s_{1} \vee e v=s_{2} \vee e v=s_{3}
\end{array}\right.
$$

O comportamento dinâmico do sistema é definido pela observação contínua do estado corrente do sistema $s=$ $\left(n_{1}, n_{2}, n_{3}, l_{2}^{n}, l_{3}^{n}, e v\right) \in S$, e pela ação adotada $a \in A(s)$ cada vez que este estado se altera, ou seja, quando ocorre um novo evento. Admite-se que a decisão é tomada imediatamente após a observação da ocorrência do evento.

Se o estado $s=\left(n_{1}, n_{2}, n_{3}, l_{2}^{n}, l_{3}^{n}, e v\right) \in S$ é observado e uma ação $a \in A(s)$ é escolhida, o sistema deve passar imediatamente a um "estado" reagido $s_{r}$ apresentado na Tabela I, e aguardar a ocorrência do próximo evento, quando ocorrerá a próxima transição. Se o último evento em $s \in S$ é a chegada de uma chamada e é tomada a decisão de aceitá-la, o sistema imediatamente muda para um "estado" reagido $s_{r}$ em que a nova chamada é incorporada ao sistema. Se o último evento é o término de uma chamada, a única ação possível é não fazer nada e a chamada deve ser imediatamente retirada do sistema, deve-se ainda fazer a redistribuição de largura de banda para as conexões da classe $j$, objetivando manter sempre o 
nível de degradação $l_{j}^{n}$ mínimo para o novo estado reagido $s_{r}$, onde $j=(2,3)$. As condições do estado $s \in S$, a ação tomada $a \in A(s)$ e o estado reagido $s_{r}$, são apresentados na Tabela I.

\section{TABELA I}

ESTADO REAGIDO.

\begin{tabular}{|c|c|c|}
\hline Condição & Ação & Estado Reagido \\
\hline $\begin{array}{l}\left(e v=a_{1}\right) \wedge \\
\left(n_{1}+1\right) b_{1}+n_{2}\left(b_{2}^{\max }-l_{2}^{n^{\prime}} \delta\right) \\
+n_{3}\left(b_{3}^{\max }-l_{3}^{n^{\prime}} \delta\right) \leq B\end{array}$ & A & $\left(n_{1}+1, n_{2}, n_{3}, l_{2}^{n \prime}, l_{3}^{n \prime}\right)$ \\
\hline $\begin{array}{l}\left(e v=a_{2}\right) \wedge \\
\left(n_{1} b_{1}+\left(n_{2}+1\right)\left(b_{2}^{\max }-l_{2}^{n^{\prime}} \delta\right)\right. \\
\quad+n_{3}\left(b_{3}^{\max }-l_{3}^{n^{\prime}} \delta\right) \leq B\end{array}$ & A & $\left(n_{1}, n_{2}+1, n_{3}, l_{2}^{n \prime}, l_{3}^{n \prime}\right)$ \\
\hline $\begin{array}{l}\left(e v=a_{3}\right) \wedge \\
\quad\left(n_{1} b_{1}+n_{2}\left(b_{2}^{\max }-l_{2}^{n^{\prime}} \delta\right)+\right. \\
\quad\left(n_{3}+1\right)\left(b_{3}^{\max }-l_{3}^{n \prime} \delta\right) \leq B\end{array}$ & A & $\left(n_{1}, n_{2}, n_{3}+1, l_{2}^{n \prime}, l_{3}^{n \prime}\right)$ \\
\hline$e v=s_{1}\left(n_{3}>0\right)$ & $\mathrm{N}$ & $\left(n_{1}-1, n_{2}, n_{3}, l_{2}^{n \prime}, l_{3}^{n \prime}\right)$ \\
\hline$e v=s_{2}\left(n_{2}>0\right)$ & $\mathrm{N}$ & $\left(n_{1}, n_{2}-1, n_{3}, l_{2}^{n \prime}, l_{3}^{n \prime}\right)$ \\
\hline$e v=s_{3}\left(n_{3}>0\right)$ & $\mathrm{N}$ & $\left(n_{1}, n_{2}, n_{3}-1, l_{2}^{n \prime}, l_{3}^{n \prime}\right)$ \\
\hline Caso contrário & $\mathrm{R}$ & $\left(n_{1}, n_{2}, n_{3}, l_{2}^{n \prime}, l_{3}^{n}\right)$ \\
\hline
\end{tabular}

Na Tabela II apresentam-se o próximo estado e a taxa de cada transição, em função do estado reagido a que chega o sistema e do próximo evento a ocorrer. Nota-se que as transições possíveis são combinações das linhas das Tabelas I e II.

TABELA II

PRÓXIMO ESTADO E TRANSIÇÃO ASSOCIADA.

\begin{tabular}{|c|c|c|}
\hline $\begin{array}{c}\text { Próximo } \\
\text { Evento }\end{array}$ & Próximo Estado & $\begin{array}{c}\text { Taxa de } \\
\text { Transição }\end{array}$ \\
\hline$a_{1}$ & $\left(t_{1}, t_{2}, t_{3}, a_{1}\right)$ & $\lambda_{1}$ \\
\hline$a_{2}$ & $\left(t_{1}, t_{2}, t_{3}, a_{2}\right)$ & $\lambda_{2}$ \\
\hline$a_{3}$ & $\left(t_{1}, t_{2}, t_{3}, a_{3}\right)$ & $\lambda_{3}$ \\
\hline
\end{tabular}

\begin{tabular}{|c|c|c|}
\hline$s_{1}$ & $\left(t_{1}, t_{2}, t_{3}, s_{1}\right)$ & $t_{1} \mu_{1}$ \\
\hline$s_{2}$ & $\left(t_{1}, t_{2}, t_{3}, s_{2}\right)$ & $t_{2} \mu_{2}$ \\
\hline$s_{3}$ & $\left(t_{1}, t_{2}, t_{3}, s_{3}\right)$ & $t_{3} \mu_{3}$ \\
\hline
\end{tabular}

\subsection{Função Retorno.}

Para completar a descrição do Processo Markoviano de Decisão (MPD), é necessário definir uma função de retorno $\mathrm{R}(\mathrm{s}, \mathrm{a})$, que corresponde ao retorno total esperado do sistema até o próximo instante de observação, dado que sistema é observado no estado $\mathrm{s}=\left(n_{1}, n_{2}, n_{3}, l_{2}^{n}, l_{3}^{n}, e v\right) \in$ $\mathrm{S}$, e a ação tomada a $\in \mathrm{A}(\mathrm{s})$ é escolhida.

No modelo apresentado, este retorno corresponde ao número de chamadas sendo transmitidas no estado corrente, eventualmente ponderados por pesos $r_{1}, r_{2}, r_{3}$ atribuídos ao número de conexões de cada uma da classe $\mathrm{i}$, $\mathrm{i}=\in\{1,2,3\}$. Como imediatamente após a escolha da ação, o sistema passa ao "estado" reagido $s_{r}=\left(t_{1}, t_{2}, t_{3}, l_{2}^{n \prime}, l_{3}^{n \prime}, e v_{r}\right)$ mostrado na Tabela 1 , é em função do estado reagido que se deve obter o retorno $\mathrm{R}(\mathrm{s}, \mathrm{a})$ da seguinte maneira:

$$
R(s, a)=\left(r_{1} t_{1}+r_{2} t_{2}+r_{3} t_{3}\right) \tau(s, a)
$$

Onde $\tau(\mathrm{s}, \mathrm{a})$ é o tempo esperado até o próximo instante de decisão, que é dado por $\tau(\mathrm{s}, \mathrm{a})=\sum_{\mathrm{s} \neq \mathrm{s}^{\prime}} \Lambda_{\mathrm{ss}^{\prime}}(\mathrm{a}) \Lambda_{\mathrm{ss}^{\prime}}(\mathrm{a}) \cdot \Lambda_{\mathrm{ss}^{\prime}}$ (a) é a taxa de transição do estado $s \in S$ para o estado $s^{\prime} \in S$, quando a ação $\mathrm{a} \in \mathrm{A}(\mathrm{s})$ é escolhida.

\section{SimulÇÕES E RESULTADOS}

Na simulação considerou-se além do conjunto de dados apresentados na tabela III, que as taxa de processamento das chamadas são $\mu_{1}=\mu_{2}=\mu_{3}=1$; e os retorno atribuídos às classes, $r_{1}=4 ; r_{2}=3 ; r_{3}=1$; Nota-se que a atribuição de diferentes retornos a cada classe de serviço é uma forma de fazer o tratamento diferenciado de cada fluxo, assim atribuí-se um retorno maior à classe com que se deseja priorizar.

Para obter uma avaliação do modelo com relação ao número de chamadas no sistema variou-se a taxa de chegadas de conexões nas classes, fazendo, $\lambda_{1}=\lambda_{2}=\lambda_{3}=$ $5,8,10,15$ e 20; Os resultados obtidos são listados na Tabela IV.

Observando os resultados da tabela IV, verifica-se quando há baixa carga no sistema (taxa de chegada de conexões) o throughput da rede é quase $100 \%$ para todas ou seja as conexões que chegam não são bloqueadas pois a rede dispõe de recursos para suprir a demanda de banda. Entretanto, conforme elevamos a carga do sistema 
$\left(\lambda_{1}=\lambda_{2}=\lambda_{3}=8,10\right)$ observa-se as há favorecimento das classes com maior retorno (classe 1,2 ), em detrimento da classe 3, com menor retorno. Conforme o sistema é alimentado com cargas ainda mais altas $\left(\lambda_{1}=\lambda_{2}=\right.$ $\left.\lambda_{3}=15,20\right)$ o favorecimento das classes com maior retorno se torna mais evidente. Ou seja, conforme os recursos se tornam insuficientes para atender a demanda total a política do CAC passa aceitar a classe com maior retorno como a classe 1 , considerada mais relevante para o sistema e rejeitar as classes com menor retorno como a classe 3 .

TABELA III

PARÂMETROS DO SISTEMA.

\begin{tabular}{|c|c|}
\hline Parâmetro & Valor \\
\hline $\mathrm{B}$ & $6 \mathrm{Mbps}$ \\
\hline$b_{1}$ & $64 \mathrm{Kbps}$ \\
\hline$b_{2}^{\min }$ & $64 \mathrm{Kbps}$ \\
\hline$b_{2}^{\max }$ & $128 \mathrm{Kbps}$ \\
\hline$b_{3}^{\min }$ & $32 \mathrm{Kbps}$ \\
\hline$b_{3}^{\max }$ & $128 \mathrm{Kbps}$ \\
\hline$\delta$ & $32 \mathrm{Kbps}$ \\
\hline
\end{tabular}

TABELA IV

RETORNO E THROUGHPUT DO SISTEMA.

\begin{tabular}{|c|c|c|c|}
\hline $\begin{array}{c}\text { Taxa de } \\
\text { Chegada }\end{array}$ & Retorno & $\begin{array}{c}\text { Classe de } \\
\text { Serviço }\end{array}$ & Throughput \\
\hline \multirow{2}{*}{5} & 20 & 1 & 5 \\
& 15 & 2 & 5 \\
& 4.99276 & 3 & 4.99276 \\
\hline \multirow{3}{*}{8} & 31.9297 & 1 & 7.98242 \\
& 23.9473 & 2 & 7.98242 \\
& 7.65386 & 3 & 7.65386 \\
\hline \multirow{3}{*}{10} & 39.053 & 1 & 9.76324 \\
& 29.2897 & 2 & 9.76324 \\
& 8.9177 & 3 & 8.9177 \\
\hline \multirow{2}{*}{15} & 54.4042 & 1 & 13.601 \\
& 36.6795 & 2 & 12.2265 \\
& 4.72091 & 3 & 4.72091 \\
\hline
\end{tabular}

\begin{tabular}{|c|c|c|c|}
\hline \multirow{3}{*}{20} & 68.1356 & 1 & 17.0339 \\
& 36.8643 & 2 & 12.2881 \\
& 0.000363702 & 3 & 0.000363702 \\
\hline
\end{tabular}

\section{CONCLUSÕES}

Neste trabalho, propôs-se um modelo de decisão markoviano para uma rede IEEE 802.16 PMP, com o objetivo de encontrar uma política de controle de admissão de conexão que maximizasse a utilização da rede. Observou-se, que a admissão de tráfegos com retornos diferenciados permite o tratamento diferenciado das classes de serviços, permitindo que em caso de grande carga no sistema as classes consideradas mais relevantes do ponto de vista do usuário sejam favorecidas. A política ótima priorizou as conexões UGS, ertPS e rtPS em detrimento das conexões da classe nrtPS.

Para trabalhos futuros, pretende-se utilizar outras funções retorno, de forma que seja possível avaliar a política ótima considerando aspectos como o nível de degradação médio das classes de serviço com relação à utilização dos recursos, procurando ainda obter políticas de controle "justas", que são aquelas que dão a mesma probabilidade de bloqueio para todas as classes de usuários.

\section{REFERÊNCIAS}

[1] IEEE 802.16e Working Group, 2005, IEEE 802.16-2005 (Revisão do IEEE 802.16-2004): IEEE Standard for Local and Metropolitan Area Networks Part 16: Air Interface for Fixed and Mobile Broadband Wireless Access Systems., IEEE Press.

[2] IEEE 802.16d Working Group, 2004, IEEE Std 802.16-2004 (Revisão do IEEE 802.16-2001): IEEE Standard for Local and Metropolitan Area Networks Part 16: Air Interface for Fixed Broadband Wireless Access Systems., IEEE Press.

[3] Frederick S. Lai, Jelena MiSiC and Samuel T. Chanson., Complete Sharing versus Partitioning: Quality of Service Management for Wireless Multimedia Networks. In Proceedings of the international Conference on Computer Communications and Networks (October 12 - 15, 1998). IC3N. IEEE Computer Society, Washington, DC, 584.

[4] Puterman, M. L. Markov Decision Processes. New York:Wiley, 1994.

[5] Haitang, W., Bing, H., Dharma, A., Above packet level admission control and bandwidth allocation for IEEE 802.16 wireless MAN. In Proceedings of the 12th international Conference on Parallel and Distributed Systems - Volume 1 (July 12 15, 2006). ICPADS. IEEE Computer Society, Washington, DC, 599-604 